\title{
Simplified Hand Configuration for Object Manipulation
}

\author{
Salvador Cobos, Manuel Ferre, Javier Ortego, and Miguel Angel Sanchéz-Urán \\ Universidad Politécnica de Madrid, \\ C/. José Gutierrez Abascal, 2, \\ 28006, Madrid, Spain \\ cobosalvador@etsii.upm.es, m.ferre@upm.es, \\ jortego@etsii.upm.es, miguelangel.sanchezurar@upm.es \\ http://www.disam.upm.es
}

\begin{abstract}
This work is focused on obtaining realistic human hand models that are suitable for manipulation tasks. Firstly, a 24 DOF kinematic model of the human hand is defined. This model is based on the human skeleton. Intra-finger and inter-finger constraints have been included in order to improve the movement realism. Secondly, two simplified hand descriptions (9 and 6 DOF) have been developed according to the constraints predefined. These simplified models involve some errors in reconstructing the hand posture. These errors are calculated with respect to the 24 DOF model and evaluated according to the hand gestures. Finally, some criteria are defined by which to select the hand description best suited to the features of the manipulation task.
\end{abstract}

Keywords: Hand model, manipulation model, finger constraints.

\section{Introduction}

The high number of DoFs (usually more than 20 DoFs) for defining the hand posture requires a longer computational time for applications that have to process this information; however, real time applications, such as gesture recognition, dynamics calculations, check collisions, etc., requires simplified models in order to obtain data faster. It is thus necessary to reduce the number of elements that make up the original hand description to fewer elements. The uncertainty of postures is not relevant for some kind of applications that can be use simplified hand descriptions in order to apply their corresponding calculations faster. Different models of the human hand currently exist. [1] describes a hand model with 26 DoF, 2 describes a hand model with 23 DoF, 3], 4. proposes a hand model with 20 DoF plus $2 \mathrm{DOF}$ for the wrist and $2 \mathrm{DoF}$ for the arm, and 5] proposes a hand model with 26 DoF. A human hand model of 24 DoFs is described in the next section. This model represents a balance between complexity and realism. It is important to indicate that more or less DoF can result in increased complexity or a decreased range of movement. Therefore, a suitable kinematic model is needed in order to conserve all the kinematic information for object manipulation. A 
significant simplification is done in the original hand description. Therefore, the most relevant finger relations and constraints have been analyzed in order to define new simplified hand models. These constraints have been obtained from literature and experiments that demonstrate the strong finger coupling in manipulation. As result, two new simplified hand model descriptions with 9 and 6 DoFs have been defined. These models represent a significant reduction in the processed information. Finally, some experiments have been carried out in order to evaluate the position errors that involve these simplified hand model descriptions. A Cyberglove [6] has been used for evaluating the error of the simplified hand description versus the full 24 DoFs hand model. Intra-finger and inter-finger constraints have been checked with the information provided by the glove. These experiments compare finger trajectories for circular and prismatic grasping by using 24, 9 and 6 DoFs.

\section{Kinematic Human Hand Model}

The hand model used for this work is based on the human skeleton. The kinematic model is comprised of 20 links that imitate the corresponding human bones, and 24 degrees of freedom (DoF) that represent the joints. Two kinematic configurations are considered in this model, one for the thumb and other for the rest of the fingers. Figure 1 shows details of this kinematics models. Main points of this model are the use of four DoF for the thumb finger modelling, the inclusion of the CMC joint and the movement concatenation in the MCP joint. The MCP abduction/adduction turn is defined first than the MCP flexion in order to better simulate finger displacements. The CMC joint allows simulating the palm arc; it represents the deformation in the palm when the hand is grasping
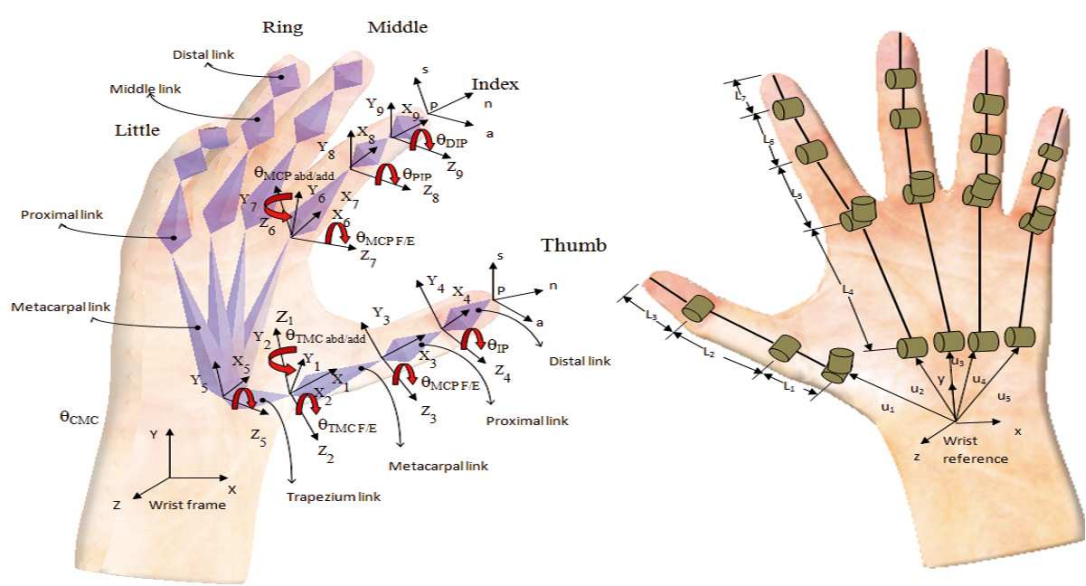

Fig. 1. Kinematic configuration of the human hand. Thumb is defined by 4 links and 4 degrees of freedom whereas index, middle, ring and little are defined by 4 links and 5 DoFs. 
Table 1. D-H Parameters for the index, middle, ring and little fingers

\begin{tabular}{|c|c|c|c|c|}
\hline Joint & $\theta_{i}$ & $d_{i}$ & $a_{i}$ & $\alpha_{i}$ \\
\hline 1 & $\theta_{C M C}$ & 0 & $L_{4}$ & $\pi / 2$ \\
2 & $\theta_{M C P a b d / a d d}$ & 0 & 0 & $-\pi / 2$ \\
3 & $\theta_{M C P F / E}$ & 0 & $L_{5}$ & 0 \\
4 & $\theta_{P I P}$ & 0 & $L_{6}$ & 0 \\
5 & $\theta_{D I P}$ & 0 & $L_{7}$ & 0 \\
\hline
\end{tabular}

Table 2. D-H Parameters for the Thumb model

\begin{tabular}{|c|c|c|c|c|}
\hline Joint & $\theta_{i}$ & $d_{i}$ & $a_{i}$ & $\alpha_{i}$ \\
\hline 1 & $\theta_{T M C a b d / a d d}$ & 0 & 0 & $\pi / 2$ \\
2 & $\theta_{T M C F / E}$ & 0 & $L_{1}$ & 0 \\
3 & $\theta_{M C P F / E}$ & 0 & $L_{2}$ & 0 \\
4 & $\theta_{I P}$ & 0 & $L_{3}$ & 0 \\
\hline
\end{tabular}

a ball or similar objects. The above points contribute to define high realistic hand movements and gestures.

\section{$2.1 \quad$ Direct Kinematics}

Direct kinematics of the index, middle, ring and little fingers. DenavitHartenberg (D-H) parameters [7] have been used for defining index, middle, ring and little finger configurations. The four fingers have four bones: metacarpal, proximal, middle and distal. These bones represent the length of each link of the serial kinematic chain. The corresponding joints to these fingers are: carpometacarpal (CMC) joint, metacarpophalangeal (MCP) joint, proximal interphalangeal (PIP) joint and distal interphalangeal (DIP) joint. The MCP joint has 2 degrees of freedom that define the adduction/abduction and flexion/extension movements. The CMC, PIP and DIP joints are of the flexion/extension type with respect to the sagittal plane of the hand. Table 1 shows the D-H parameters for index, middle, ring and little fingers.

Direct kinematics of the thumb. The thumb has been modeled by four principal bones: trapezium, metacarpal, proximal and distal. The joints corresponding to these fingers are: trapeziometacarpal (TMC), metacarpophalangeal (MCP) and interphalangeal (IP) joints. The TMC joint has 2 DoF in Flexion/Extension and Adduction/Abduction. Table 2 shows the D-H parameters for the thumb model.

\section{Dynamic Constraints of Finger Movements}

In this paper, new constraints have been defined in the inter-finger and intrafinger categories, so as to obtain more realistic movements in the hand model. Intra-finger constraints have been developed to reproduce movements of finger trajectories such as circular and prismatic grasps [8]. Inter-finger constraints have been verified in experiments carried out with a Cyberglove [9] to obtain the dependency of tendons mainly among the middle, ring and little fingers.This type of constraint refers to some dependency among fingers while they are in 
motion [10]. The inter-finger constraints were obtained by using the hand model and Cyberglove. These types of constraints are coupled movements among the index, middle, ring and little fingers. The relationship among angles with the middle, ring and little fingers has been measured to represent real movements of the hand model. The Cases of involuntary movements have been defined in 11. Middle, ring and little fingers share common flexor tendons. It implies involuntary movements due to this strong coupling.

\subsection{Simplified Hand Description for Circular Grasping}

Circular grasping implies a strong relation among finger joints. These relations are summarized in the Table 3. The most accepted intra-finger constraint is: $\theta_{D I P}=\frac{2}{3} \theta_{P I P}$. It has been analyzed by several researchers, such as 12 and has been efficiently checked in our experiments. According to table 3 a significant reduction can be done in the number of DoF used for defining the grasping gesture. 8 DoFs $\left(\theta_{T M C(a b d / a d d)}, \theta_{I P-T}, \theta_{M C P(a b d / a d d)-I}, \theta_{D I P-I}, \theta_{D I P-M}, \theta_{C M C-L}\right.$, $\left.\theta_{M C P(a b d / a d d)-L}, \theta_{D I P-L}\right)$ have been considered for defining a circular grasping gesture. The rest of the $24 \mathrm{DoFs}$ defined in section 2 are obtained according to the table 3. Main conclusions of this table are that thumb is defined by 2 DoFs, index by 2 DoFs, middle by 1 DoF and little by 3 DoFs. The ring is calculated by the little and middle joints. Therefore, the thumb, the index and the little are the most important fingers when defining circular gestures.

Table 3. Intra-finger constraints for circular grasping

\begin{tabular}{|c|c|c|c|c|}
\hline Thumb & Index & Middle & Ring & Little \\
\hline$\theta_{T M C a b d / a d d}$ & $\theta_{C M C-I}=$ & $\theta_{C M C-M}=$ & $\theta_{C M C-R}=$ & $\theta_{C M C-L}$ \\
& $\theta_{C M C-M}$ & $\frac{1}{2} \theta_{C M C-R}$ & $\frac{2}{3} \theta_{C M C-L}$ & \\
\hline$\theta_{T M C F / E}=$ & $\theta_{M C p a b d / a d d-I}$ & $\theta_{M C P a b d / a d d-M}=$ & $\theta_{M C P a b d / a d d-R}=$ & $\theta_{M C P a b d / a d d-L}$ \\
$\frac{11}{10} \theta_{M C P}$ & & $\frac{1}{5} \theta_{M C P a b d / a d d-I}$ & $\frac{1}{2} \theta_{M C P a b d / a d d-L}$ & \\
\hline$\theta_{M C P}=$ & $\theta_{M C P F / E-I}=$ & $\theta_{M C P F / E-M}=$ & $\theta_{M C P F / E-R}=$ & $\theta_{M C P F / E-L}=$ \\
$\frac{4}{5} \theta_{I P}$ & $\frac{4}{3} \theta_{P I P-I}$ & $\frac{4}{3} \theta_{P I P-M}$ & $\frac{4}{3} \theta_{P I P-R}$ & $\frac{4}{3} \theta_{P I P-L}$ \\
\hline$\theta_{I P}$ & $\theta_{P I P-I}=$ & $\theta_{P I P-M}=$ & $\theta_{P I P-R}=$ & $\theta_{P I P-L}=$ \\
& $\frac{3}{2} \theta_{D I P-I}$ & $\frac{3}{2} \theta_{D I P-M}$ & $\frac{3}{2} \theta_{D I P-R}$ & $\frac{3}{2} \theta_{D I P-L}$ \\
\hline & $\theta_{D I P-I}$ & $\theta_{D I P-M}$ & $\theta_{D I P-R}$ & $\theta_{D I P-L}$ \\
\hline
\end{tabular}

\subsection{Simplified Hand Description for Prismatic Grasping}

Prismatic grasping represents a less constraint among finger that circular grasping. In this case 9 DoFs $\left(\theta_{T M C(a b d / a d d)-T}, \theta_{I P-T}, \theta_{M C P(a b d / a d d)-I}, \theta_{D I P-I}\right.$, $\left.\theta_{D I P-M}, \theta_{D I P-R}, \theta_{C M C-L}, \theta_{M C P(a b d / a d d)-L}, \theta_{D I P-L}\right)$ are used for defining a prismatic grasping gesture. The rest of the 24 DoFs defined in section 2 are obtained according to the table 4. Main conclusions of this table are that thumb is defined by 2 DoFs, index by 2 DoFs, middle by 1 DoF and little by 3 DoFs. 
Table 4. Intra-finger constraints for prismatic grasping

\begin{tabular}{|c|c|c|c|c|}
\hline Thumb & Index & Middle & Ring & Little \\
\hline$\theta_{T M C a b d / a d d}$ & $\theta_{C M C-I}=$ & $\theta_{C M C-M}=$ & $\theta_{C M C-R}=$ & $\theta_{C M C-L}$ \\
& $\theta_{C M C-M}$ & $\frac{1}{2} \theta_{C M C-R}$ & $\frac{2}{3} \theta_{C M C-L}$ & \\
\hline$\theta_{T M C F / E}=$ & $\theta_{M C p a b d / a d d-I}$ & $\theta_{M C P a b d / a d d-M}=$ & $\theta_{M C P a b d / a d d-R}=$ & $\theta_{M C P a b d / a d d-L}$ \\
$\frac{10}{11} \theta_{M C P}$ & & $\frac{1}{5} \theta_{M C P a b d / a d d-I}$ & $\frac{1}{2} \theta_{M C P a b d / a d d-L}$ & \\
\hline$\theta_{M C P}=$ & $\theta_{M C P F / E-I}=$ & $\theta_{M C P F / E-M}=$ & $\theta_{M C P F / E-R}=$ & $\theta_{M C P F / E-L}=$ \\
$\frac{6}{5} \theta_{I P}$ & $\frac{3}{2} \theta_{P I P-I}$ & $\frac{3}{2} \theta_{P I P-M}$ & $\frac{3}{2} \theta_{P I P-R}$ & $\frac{3}{2} \theta_{P I P-L}$ \\
\hline$\theta_{I P}$ & $\theta_{P I P-I}=$ & $\theta_{P I P-M}=$ & $\theta_{P I P-R}=$ & $\theta_{P I P-L}=$ \\
& $2 \theta_{D I P-I}$ & $2 \theta_{D I P-M}$ & $2 \theta_{D I P-R}$ & $2 \theta_{D I P-L}$ \\
\hline & $\theta_{D I P-I}$ & $\theta_{D I P-M}$ & $\theta_{D I P-R}$ & $\theta_{D I P-L}$ \\
\hline
\end{tabular}

Table 5. Error indixes among original hand model and hand posture reconstructed

\begin{tabular}{|c|c|c|}
\hline & Error circular grasping \% & Error prismatic grasping \% \\
\hline Original model (24 DOF) & - & - \\
\hline 9 DoF hand description & $7.67 \%$ & $5.90 \%$ \\
\hline 6 DoF hand description & $13.14 \%$ & $9.18 \%$ \\
\hline
\end{tabular}

\section{Experiments of the Simplified Hand Models}

These experiments have been carried out for circular and precision postures. Each hand description is used for reconstructing the corresponding posture (circular and prismatic). Table 5 contains error indexes of each hand description. These error indexes were obtained measuring the error between the fingertip positions of the posture reconstructed with respect to original model of $24 \mathrm{DOF}$. Cyberglove and hand model were used to obtain the original posture that correspond to the gesture to reconstructing. It is important to indicate that these results depend on the user calibration. Data can vary 1-2\% among users according to their hand size. The optimum number of DoFs for describing hand postures depends on the precision/simplification relation of each application. Therefore it is required to achieve a balance between the maximum acceptable error and minimum hand DoFs to process. According to results shown in table 5 , applications that admit errors close to $10 \%$ can use 6 DoF hand models, more accurate precisions between 5-10\% errors require 9 DoFs for hand descriptions. Finally, if positions errors must to be less than $5 \%$ then models with more than 20 DoFs have to be used.

\section{Conclusions}

Three human hand models have been analyzed in this work. The hand model with 24 DoFs is appropriated for very realistic manipulations. This model has also been used to obtain new Dynamic constraints. These constraints permit allow obtaining another simplified hand descriptions. Two simplified hand 
descriptions with 9 and 6 DoFs have been proposed and evaluated. Dynamic constraints are used to estimate the rest of finger joints up to the original 24 components. These models represent a significant reduction in the number components used to describe the hand gesture. Experiments carried out for both simplified hand descriptions show that position errors of the fingertip are close to $6 \%$ for $9 \mathrm{DoF}$ and $13 \%$ for $6 \mathrm{DoF}$.

Acknowledgments. This work is supported by the European commission under the IMMERSENCE integrated project of the sixth frame program (IST-4027141-IP). Also we thank to CONACYT by the supports offered to Salvador Cobos.

\section{References}

1. Bray, M., Koller-Meier, E., Müller, P., Schraudolph, N.N., Van Gool, L.: Stochastic optimization for high-dimensional tracking in dense range maps. In: Proc. IEEE. Vis. Image Signal Process, pp. 501-512 (August 2005)

2. Kuch, J.J., Huang, T.S.: Human Computer Interaction via the Human Hand: A Hand Model. In: Signals, systems and Computers, Conference Record of the Twenty Eighth Asilomar Conference, pp. 1252-1256 (1994)

3. Chalfoun, J., Renault, M., Younes, R., Ouezdou, F.B.: Muscle Forces prediction of the Human Hand and Forearm system in Highly Realistic Simulation. In: Proc. IEEE/RSJ International Conference on Intelligent Robots and Systems, pp. 1293$1298(2004)$

4. Renault, M., Oezdou, F.B.: Dynamic simulation of hand-forearm system. In: IEEE International Workshop on Robot and Human Interactive Communication, pp. 20-25 (2001)

5. Du, H., Charbon, E.: 3d Hand Model Fitting for Virtual Keyboard System. In: IEEE workshop on Applications of Computer Vision, pp. 31-36 (February 2007)

6. http://www.virtex.com

7. Denavit, J., Hartenberg, R.S.: A Kinematic Notation for Lower-Pair Mechanisms Based on Matrices. Journal of Applied Mechanics 77, 215-221 (1955)

8. Cutkosky, M.R., Wright, P.K.: Modeling Manufacturing grips and correlations with design of robotic hands. In: Procedings of the IEEE International Conference on Robotics and Automation, pp. 1533-1539 (1986)

9. Virtual Technologies. Reference Manual. CG081998-2-1 (1998)

10. Lin, J., Wu, Y., Huang, T.S.: Modeling the Constrains of Human Hand Motion. In: IEEE Human Motion Proceedings, pp. 121-126 (2000)

11. Cobos, S., Ferre, M., Sanchéz-Urán, M.A., Ortego, J.: Constraints for Realistic Hand Manipulation. In: PRESENCE 2007, The 10th Annual International Workshop on Presence, Barcelona, Spain, October 25-27 (2007)

12. Fahn, C.S., Sun, H.: Development of a Data Glove with Reducing Sensors Based on Magnetic Induction. IEEE Transactions on Industrial Electronics 52(2) (2005) 